\title{
Genomic discovery of the hypsin gene and biosynthetic pathways for terpenoids in Hypsizygus marmoreus
}

Byoungnam Min ${ }^{1 \dagger}$, Seunghwan $\mathrm{Kim}^{2 \dagger}$, Youn-Lee $\mathrm{Oh}^{3}$, Won-Sik Kong ${ }^{3}$, Hongjae Park', Heejung Cho², Kab-Yeul Jang ${ }^{3}$, Jeong-Gu Kim² ${ }^{2^{*}}$ and In-Geol Choi ${ }^{1^{*}}$ (i)

\begin{abstract}
Background: Hypsizygus marmoreus (Beech mushroom) is a popular ingredient in Asian cuisine. The medicinal effects of its bioactive compounds such as hypsin and hypsiziprenol have been reported, but the genetic basis or biosynthesis of these components is unknown.

Results: In this study, we sequenced a reference strain of $H$. marmoreus (Haemi 51,987-8). We evaluated various assembly strategies, and as a result the Allpaths and PBJelly produced the best assembly. The resulting genome was $42.7 \mathrm{Mbp}$ in length and annotated with 16,627 gene models. A putative gene (Hypma_04324) encoding the antifungal and antiproliferative hypsin protein with $75 \%$ sequence identity with the previously known N-terminal sequence was identified. Carbohydrate active enzyme analysis displayed the typical feature of white-rot fungi where auxiliary activity and carbohydrate-binding modules were enriched. The genome annotation revealed four terpene synthase genes responsible for terpenoid biosynthesis. From the gene tree analysis, we identified that terpene synthase genes can be classified into six clades. Four terpene synthase genes of $\mathrm{H}$. marmoreus belonged to four different groups that implies they may be involved in the synthesis of different structures of terpenes. A terpene synthase gene cluster was well-conserved in Agaricomycetes genomes, which contained known biosynthesis and regulatory genes.
\end{abstract}

Conclusions: Genome sequence analysis of this mushroom led to the discovery of the hypsin gene. Comparative genome analysis revealed the conserved gene cluster for terpenoid biosynthesis in the genome. These discoveries will further our understanding of the biosynthesis of medicinal bioactive molecules in this edible mushroom.

Keywords: Hypsizygus marmoreus, Beech mushroom, Fungal genome, Hypsin, Marmorin, Hypsiziprenol A9, Secondary metabolism

\section{Background}

Hypsizygus marmoreus is an edible mushroom with various medicinal effects, including antitumor, antibacterial, and antifungal properties [1-4] (Fig. 1). Several bioactive molecules have been reported to underlie the medicinal effects of H. marmoreus; in particular, the terpenoid compound hypsiziprenol A9 inhibits cell cycle progression in HepG2 cells, a human liver cancer cell line [2]. The

\footnotetext{
* Correspondence: jkim5aug@korea.kr; igchoi@korea.ac.kr

${ }^{\dagger}$ Byoungnam Min and Seunghwan Kim contributed equally to this work.

${ }^{2}$ Genomics Division, National Institute of Agricultural Sciences, Rural

Development Administration (RDA), Jeonju 54874, Korea

'Department of Biotechnology, College of Life Sciences and Biotechnology,

Korea University, 145 Anam-ro, Seongbuk-Gu, Seoul 02841, Korea
}

Full list of author information is available at the end of the article thermostable ribosome-inactivating protein hypsin, which can be extracted from the fruiting body of the mushroom, has antifungal and antiproliferative properties [5]. Another ribosome-inactivating protein, marmorin, has antiproliferative and HIV-1 reverse transcriptase inhibitory activities [6]. Despite the popularity of $H$. marmoreus as a gastronomic and medicinal resource, the genetic basis or biosynthetic pathways of these active compounds are unknown. Using genome sequencing, we aim to understand the mushroom's bioactivity at the genomic level.

Various terpenoid compounds from different mushrooms have been reported to have medicinal effects [7]. Genome sequencing methods have been used to elucidate the biosynthetic pathways of terpenoid compounds

(c) The Author(s). 2018 Open Access This article is distributed under the terms of the Creative Commons Attribution 4.0 International License (http://creativecommons.org/licenses/by/4.0/), which permits unrestricted use, distribution, and reproduction in any medium, provided you give appropriate credit to the original author(s) and the source, provide a link to the Creative Commons license, and indicate if changes were made. The Creative Commons Public Domain Dedication waiver (http://creativecommons.org/publicdomain/zero/1.0/) applies to the data made available in this article, unless otherwise stated. 


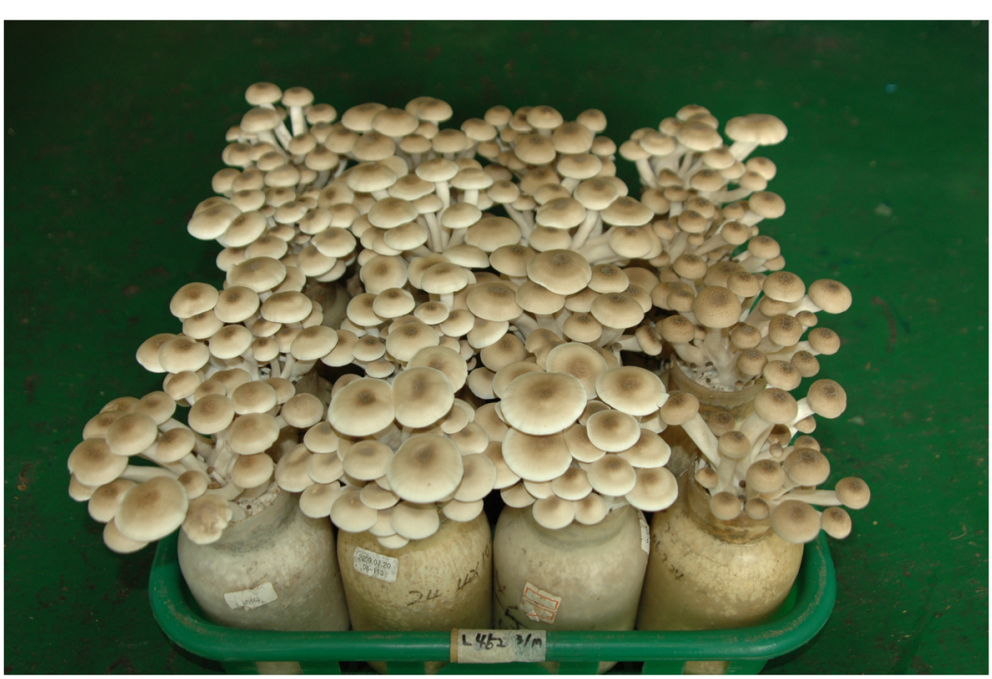

Fig. 1 Fruiting bodies of Hypsizygus marmoreus

by identifying terpene synthase genes. For example, potential terpene synthase genes in Coprinus cinereus [8], Omphalotus olearius [9], and Stereum hirsutum [10] have been mined via genome sequencing, and their biochemical activities have been studied. In particular, coexpression of cop6 and the two P450 monooxygenase genes of C. cinereus has been reported to produce the antimicrobial compound lagopodin [8]. Thus, it is important to combine molecular, genetic, and biochemical techniques within the genomic context to understand the biosynthesis of natural bioactive compounds. Both biochemical compounds and many proteins, such as lectins, fungal immunomodulatory proteins, ribosomeinactivating proteins, ribonucleases, and laccases, have been suggested as candidates for medicinally active components in mushrooms [11]. Ribosome-inactivating proteins inhibit protein synthesis by modifying ribosomal RNA. This results in HIV-1 reverse transcriptase inhibition as well as antifungal, anticancer, and antiproliferative activities [11]. Plants are the primary sources of these ribosome-inactivating proteins [3] and some mushrooms. Examples of ribosome-inactivating proteins expressed in mushrooms include velutin (Flammulina velutipes) [12], flammulin (F. velutipes) [13], and lyophyllin
(Lyophyllum shimeji) [14]. Genes encoding these proteins have not yet been explored despite the availability of genome sequences for these species $[15,16]$.

In this study, we reported the fully annotated genome of $H$. marmoreus and elucidated the genetic basis of the biosynthesis of bioactive molecules reported in this mushroom. We obtained a high-quality genome assembled from three different sequencing libraries using multiple genome assembly strategies. Sequential and functional comparisons enabled us to identify the hypsin gene in the genome. Orthologous gene analysis identified putative genes responsible for biosynthesizing hypsiziprenol A9.

\section{Results}

Genome assembly using various strategies

We constructed and sequenced three genomic DNA sequencing libraries: paired-end and mate-pair Illumina libraries and a PacBio library (Table 1). To obtain a high-quality genome assembly, we applied five assembly strategies to the assembly procedure: (i) Allpaths, (ii) Allpaths+PBJelly, (iii) Allpaths+SSPACE-longread, (iv) Falcon, and (v) SPAdes. Allpaths assembled the two Illumina libraries, and PBJelly [17] and SSPACE-longread

Table 1 Sequencing data summary

\begin{tabular}{lllll}
\hline Type & Library & Insert size & Average & Number of total reads \\
\hline Genome & Illumina paired-end & $400 \mathrm{bp}$ & $300 \mathrm{bp}$ & $14,888,962 \times 2$ \\
& Illumina mate-pair & $5000 \mathrm{bp}$ & $100 \mathrm{bp}$ & $157,055,636 \times 2$ \\
PacBio & - & $7980 \mathrm{bp}$ & $1,125,617(6 \mathrm{cells})$ \\
Transcriptome & Illumina paired-end 1 & - & $100 \mathrm{bp}$ & $25,218,416 \times 2$ \\
& Illumina paired-end 2 & - & $100 \mathrm{bp}$ & $89,796,090 \times 2$ \\
\hline
\end{tabular}

The two transcriptome libraries are technical replicates of the same sample 
[18] individually improved the assembly with PacBio reads. SPAdes [19] used all three libraries for a hybrid assembly. Because we had over $200 \times$ physical coverage of PacBio reads, we also sequentially used Falcon [20], FinisherSC [21], and Quiver [22] for PacBio-only assembly. The results of the five assembly strategies are summarized in Table 2. From the assembly assessment, we selected the Allpaths+PBJelly assembly for further analyses (See Discussion).

The final assembly had a size of 42,710,661 bp including 235 scaffolds/278 contigs with $287.3 \times$ sequence coverage. The GC percentage was $49.64 \%$. We estimated the genome size as $43.0 \mathrm{Mbp}$ using the k-mer frequency calculation of Illumina paired-end reads (Additional file 1: Figure S1). We confirmed that the assembly was a haploid genome rather than a diploid genome from the single peak in the k-mer frequency plot (Additional file 1: Figure S1). Many mushrooms were in the dikaryon stage, which introduced diploidy into their assembly. This impedes interpretation of the genome because it is difficult to differentiate between duplication and diploidy. We analyzed the ploidy of the assembly by drawing a read coverage histogram and confirmed that the genome was monokaryotic (Additional file 1: Figure S2). This was consistent with the results of the k-mer frequency estimation. There was no obvious mitochondrial or contaminated sequence in the assembly (Additional file 1: Figure S3).

\section{Repeat elements}

To avoid spurious gene prediction due to repeats, we identified a total of 2,482,387 bp (5.81\%) interspersed repeat regions. This included 28 long interspersed nuclear element (7629 bp), 867 long terminal repeat elements (887,560 bp), 586 DNA elements (378,418 bp), and 2044 unclassified elements $(1,208,780 \mathrm{bp})$. We masked these regions for gene prediction.

\section{Genome annotation}

Using the FunGAP pipeline [23], we predicted 16,627 protein-coding genes with an average size of $1586.1 \mathrm{nt}$. Of these protein-coding genes, 14,179 genes (85.3\%) were supported by assembled transcripts, and this included 10,522 (63.3\%) highly supported genes (>90\% coverage). Genome completeness was calculated using BUSCO v3.0 at the gene level. Only 5 of 1335 singlecopy entries were missing, indicating $>99 \%$ genome completeness. The quality of the gene prediction was evaluated by comparing the predictions of three programs inside the FunGAP pipeline: Augustus 3.2.1 [24], Braker 1.8 [25], and Maker 2.31.8 [26] (Additional file 2: Table S1). Gene prediction results are summarized in Table 3.

Approximately half of the predicted genes were functionally annotated; in total, 7786 genes $(46.8 \%)$ were annotated using Pfam domains, and 7447 genes (44.8\%) were annotated using SwissProt. The dominant functions included WD, F-box, protein kinase, cytochrome P450, and major facilitator superfamily domains, similarly as observed in other mushroom genomes [27, 28]. The genome contained 1793 genes encoding secreted proteins. We identified 1262 noncoding RNA elements containing 171 tRNAs, including 9 selenocysteine tRNAs, 191 small nucleolar RNAs (snoRNAs) from 127 different families, and 224 microRNAs from 90 different families.

\section{Phylogenetic location in Agaricomycetes}

In March 2017, 85 Agaricomycetes genome assemblies with predicted genes were present in the NCBI database. We excluded 15 genomes with low BUSCO completeness (<95\%); thus, we compared our assembled $H$. marmoreus genome with 69 reference genomes (Additional file 2: Table S2). In the resulting genome tree, $H$. marmoreus

Table 2 Preliminary assemblies using five assembly strategies

\begin{tabular}{|c|c|c|c|c|c|}
\hline Metrics & Allpaths & Allpaths+PBJelly & Allpaths+SSPACE-longread & SPAdes & Falcon \\
\hline Libraries & $\begin{array}{l}\text { Paired-end } \\
\text { Mate-pair }\end{array}$ & $\begin{array}{l}\text { Paired-end } \\
\text { Mate-pair } \\
\text { PacBio }\end{array}$ & $\begin{array}{l}\text { Paired-endMate-pair } \\
\text { PacBio }\end{array}$ & $\begin{array}{l}\text { Paired-end Mate-pair } \\
\text { PacBio }\end{array}$ & PacBio \\
\hline Number of scaffolds & 340 & 235 & 150 & 199 & 59 \\
\hline Number of contigs & 1000 & 278 & 1018 & 261 & 59 \\
\hline Assembly size (Mbp) & 41.6 & 42.7 & 42.3 & 42.1 & 42.2 \\
\hline N50 value (scaffolds) & $628.3 \mathrm{kbp}$ & $764.8 \mathrm{kbp}$ & $947.1 \mathrm{kbp}$ & $1.1 \mathrm{Mbp}$ & 1.6 Mbp \\
\hline N50 value (contigs) & $152.4 \mathrm{kbp}$ & $621.3 \mathrm{kbp}$ & $149.5 \mathrm{kbp}$ & $766.3 \mathrm{kbp}$ & $1.6 \mathrm{Mbp}$ \\
\hline Number of scaffolds > $1 \mathrm{Mbp}$ (scaffold sizes sum) & 5 (8.4 Mbp) & 7 (12.9 Mbp) & 11 (18.6 Mbp) & 12 (22.8 Mbp) & 15 (29.2 Mbp) \\
\hline Complete BUSCOs & 1298 & 1304 & 1300 & 1301 & 1298 \\
\hline Fragmented BUSCOs & 24 & 20 & 22 & 21 & 22 \\
\hline Missing BUSCOs & 13 & 11 & 13 & 13 & 15 \\
\hline CGAL value & $-3.61 e+09$ & $-3.52 e+09$ & $-3.90 e+09$ & $-1.12 e+09$ & $-2.83 e+09$ \\
\hline
\end{tabular}


Table 3 Gene prediction summary

\begin{tabular}{ll}
\hline Attributes & Values \\
\hline Total protein-coding genes & 16,627 \\
Transcript length (average/median) & $1586.1 / 1316$ \\
CDS length (average/median) & $1275.5 / 1038$ \\
Protein length (average/median) & $425.2 / 346$ \\
Exon length (average/median) & $221.8 / 129$ \\
Intron length (average/median) & $65.4 / 55$ \\
Spliced genes & $14,685(88.32 \%)$ \\
Gene density (genes/Mb) & 389.29 \\
Coding regions & $49.65 \%$ \\
Number of introns & 79,007 \\
Number of introns per gene (med) & 4 \\
Number of exons & 95,634 \\
Number of exons per gene (med) & 4 \\
\hline
\end{tabular}

clustered with other Agaricales species, with Termitomyces identified as the closest relative (Fig. 2). Agaricomycetes species had genome sizes of 25-119 Mbp with 926232,854 genes. The genome size and gene number of $H$. marmoreus were close to the average of these distributions (Additional file 1: Figure S4).

\section{Carbohydrate active enzymes (CAZymes)}

The H. marmoreus genome contained a total of 630 CAZyme modules within 590 genes, including 222 glycoside hydrolases (GHs), 96 glycosyltransferases, 89 carbohydrate esterases, 21 polysaccharide lyases, 80 carbohydrate-binding modules (CBMs), and 122 auxiliary activity (AA) modules (Fig. 3a). Compared with other Agaricomycetes genomes, the $H$. marmoreus genome was enriched in AA and CBM modules $(P<0.05)$. The reference genomes had median values of $91 \mathrm{AA}$ and 55 CBM modules. In detail, five subclasses were particularly enriched in $H$. marmoreus: AA1, AA3, AA9, CBM1, and CBM13 ( $<<0.05$, Fig. 3b). AA1, AA3, and AA9 encode multicopper oxidases, glucose-methanolcholine oxidoreductases, and copper-dependent lytic polysaccharide monooxygenases, respectively. These genes are well-known lignocellulose-degrading enzymes [29]. As H. marmoreus is known as a white-rot fungus [30], these enriched CAZyme families are congruent with the representative feature of white-rot fungal genomes [31]. The cellulose-binding CBM1 module is generally enriched in white-rot fungal genomes, whereas brown-rot fungal genomes have none or a few of these modules [31]. We identified 25 CBM1 modules in the genome. These modules were found with various CAZyme modules including GH6, GH7, and AA9 in various genes, which may lead to synergetic degradation. Whereas CBM13 modules related to the ricin-type beta-trefoil lectin domain (Pfam: PF00652) are found as part of many carbohydrate-binding proteins [32-34], none of these domains were accompanied by other CAZyme modules. This suggests that CBM13-containing proteins in this genome are not involved in carbohydrate degradation processes. Instead, all these proteins were extracellular proteins. Further experimental verification is needed to reveal their biological and molecular functions. In summary, the H. marmoreus CAZyme profile revealed the features of white-rot fungi with enriched lignocellulose-degrading enzymes.

\section{Candidate hypsin and marmorin genes}

Hypsin and marmorin are two major bioactive proteins previously reported as ribosome-inactivating proteins with antiproliferative activities against tumor cells $[5,6]$. The $\mathrm{N}$-terminal sequences of both proteins are "ITFQGDLDARQQVITNADTRRKRDVRAAVR" (28 amino acids) for hypsin and "AEGTLLGSRATCESGNSMY" (19 amino acids) for marmorin. The N-terminal sequence of hypsin was similar to those of plant ribosome-inactivating proteins, such as alpha-momorcharin [35] and trichosanthin [36]. The molecular weights were 20 and $9.5 \mathrm{kDa}$ for hypsin and marmorin, respectively. We searched the entire genome for these $\mathrm{N}$-terminal sequences and identified a potential hypsin gene (Hypma_04324) with $71 \%$ identity (20/28 matches) and $75 \%$ positive matches (21/28) (Fig. 4).

\section{Secondary metabolism genes}

The $H$. marmoreus genome contained 20 secondary metabolism gene clusters, including six terpene/phytoene synthases, three type-I polyketide synthases, one siderophore synthase, one nonribosomal peptide synthase, two indole synthases, and seven unknown clusters. Agaricales genomes contained an average of 27 gene clusters (range, 14-49) (Fig. 5). Type-III polyketide synthases, which have been functionally characterized in several ascomycetes [37], were lacking in all Agaricales genomes. However, the Phanerochaete carnosa (Polyporales) and Exidia glandulosa (Auriculariales) genomes contained one copy each (Additional file 2: Table S3).

Cytochrome P450 has an important role in modifying backbone secondary metabolites, such as lanosterol [38]. The $H$. marmoreus genome contained 132 cytochrome P450 domains. The two Moniliophthora genomes contained the largest numbers of this domain (342 and 361 domains, respectively, Fig. 5). Glucan synthases produce various glucan compounds with bioactive properties. All Agaricales genomes contained 2-4 glucan synthase genes, with $H$. marmoreus containing two. Major facilitator superfamily and $\mathrm{ABC}$ transporters are important in transporting secondary metabolites [39]. We found 125 


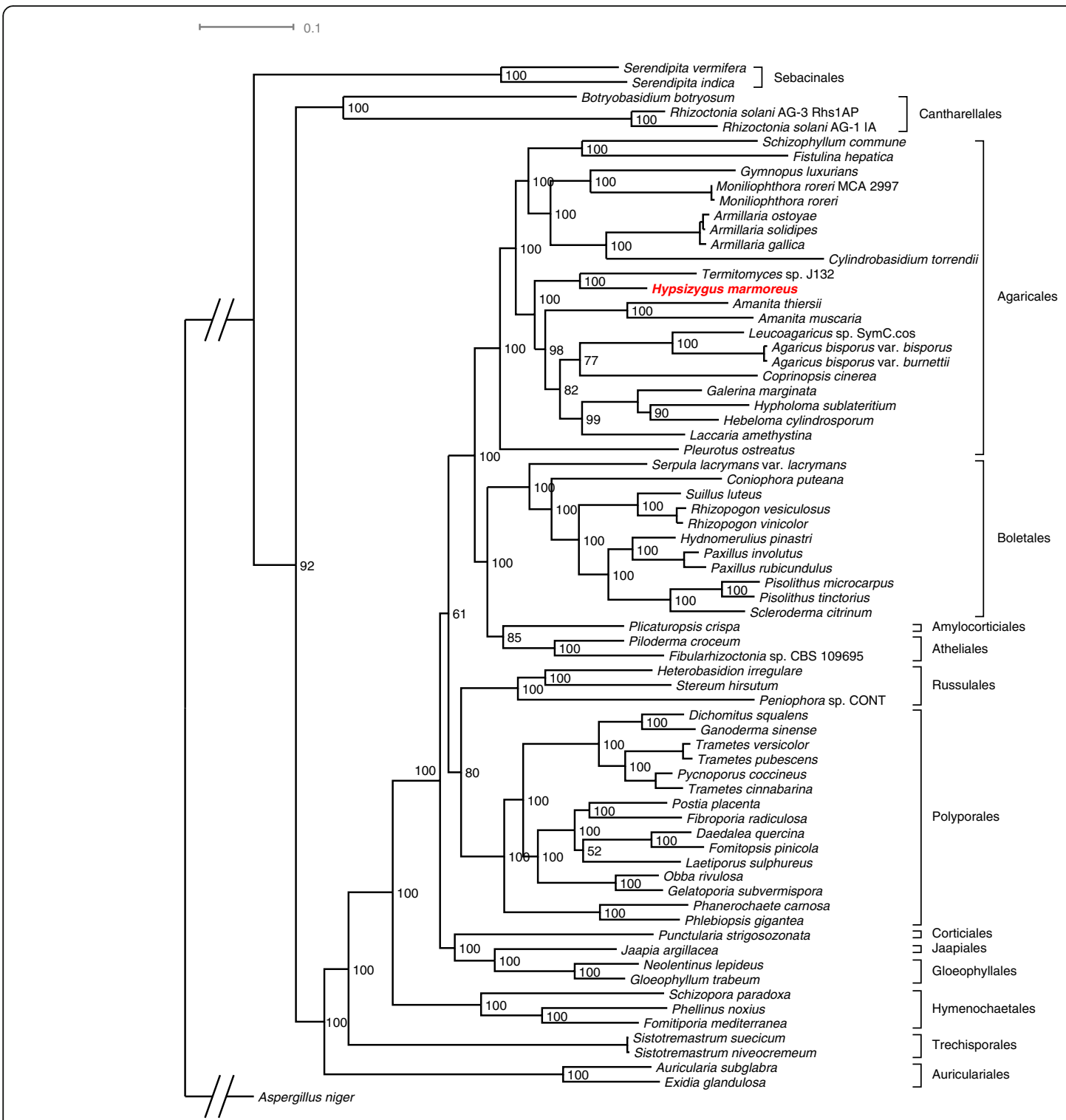

Fig. 2 Phylogenetic location of Hypsizygus marmoreus among Agaricomycetes. Phylogeny was inferred from 102 concatenated single-copy orthologs using RAxML 8.1.3. Bootstrapping-based branch support and a scale bar representing the mean number of amino acid substitutions per site are shown

and 49 major facilitator superfamily and $\mathrm{ABC}$ transporter genes, respectively, in the H. marmoreus genome.

\section{Sesquiterpene synthases}

Various terpenoid compounds are produced by biosynthetic clusters. H. marmoreus produces the terpene compound hypsiziprenol A9, which has antitumor properties [2]. To elucidate the conserved and diverged structures of terpenoid gene clusters, we obtained 759 terpene synthase genes from the 70 Agaricomycetes genomes. The Agaricomycetes genomes contained 1-25 terpene synthase genes, including four genes in the $H$. marmoreus genome (Additional file 1: Figure S5). From the gene tree, we identified six groups of terpene synthase genes, as reported previously $[8,10]$ (Fig. 6 and Additional file 1: Figure S6). The orthologs of three well-characterized C. cinereus terpene synthases, Cop1, Cop3, and Cop4, were identified in the $H$. marmoreus genome.

Various functional genes are clustered with terpene synthase genes, including terpene-modifying enzymes, regulatory proteins, and transporters (Fig. 7 and Additional file 1: Figures S7-S9). In particular, clade 6 terpene synthase genes had well-conserved gene clusters across all Agaricomycetes orders including Agaricales, Boletales, Polyporales, Russulales, and Jaapiales. This cluster contained galacto-kinase, homoserine-kinase, mevalonate-kinase, phosphomevalonate-kinase (GHMP kinase, Pfam: PF00288, PF08544), HMGL-like domain 
a

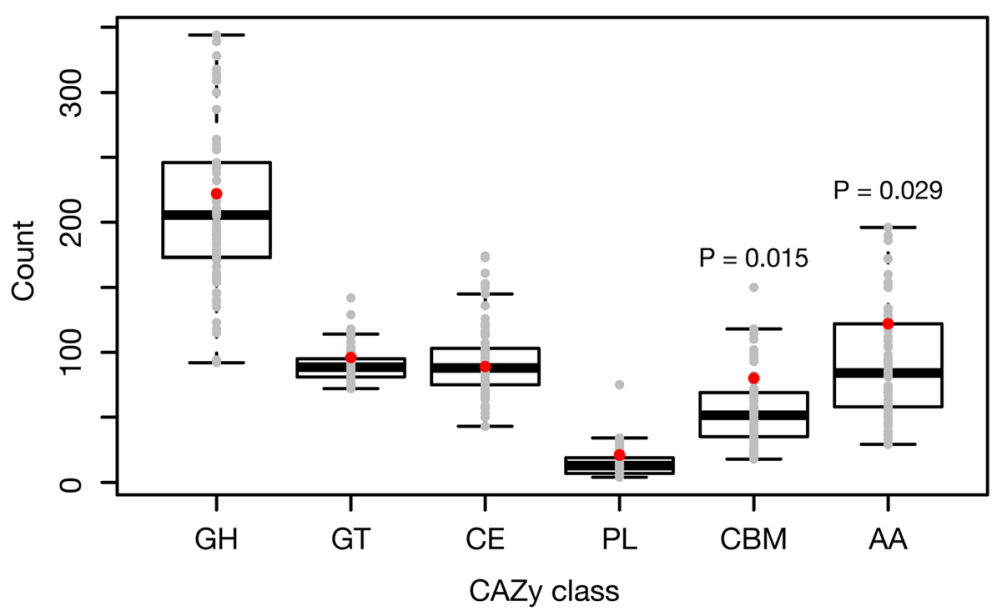

b Enriched CAZymes subclasses $(P<0.05)$

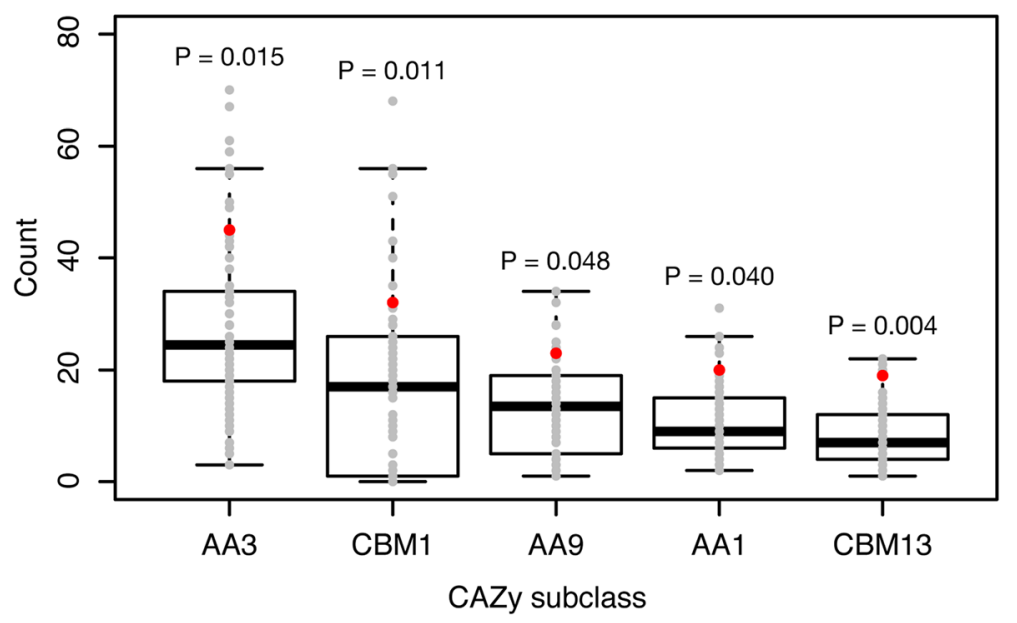

Fig. 3 Carbohydrate active enzymes (CAZymes) in Agaricomycetes genomes. a Distribution of six CAZyme classes. The $P$ values were calculated using the Scipy (https://www.scipy.org) stats.fisher_exact function, which performs Fisher's exact test. Only significant P values $(P<0.05)$ are indicated. Red points indicate the Hypsizygus marmoreus genome. b Enriched CAZyme subclasses in the H. marmoreus genome $(P<0.05)$. Significantly depleted CAZyme subclasses were not identified

(pyruvate carboxylase, Pfam: PF00682), HIT zinc finger (Pfam: PF04438), and response regulator receiver domain (Pfam: PF00072) (Fig. 7). GHMP kinase and HMGL-like domains are directly related to the biosynthesis of terpenoids [40, 41]. HIT zinc finger and response regulator receiver domains are related to gene regulation [42, 43]. These well-conserved gene clusters suggest the putative resemblance of their product structure and their regulation. Conversely, clade 5 lacked a conserved gene cluster. In other gene clusters, transporter (major facilitator superfamily), heat shock protein activator, helicase, and F-box-like domains were frequently identified. Their exact molecular functions and associations with terpene synthesis remain to be elucidated by in vitro/in vivo experiments. The terpene synthases and their adjacent genes displayed transcriptional activity in hyphae (Additional file 1: Figure S10). This suggests that they are coregulated, although further investigation is needed to reveal whether they are related to the biosynthesis of a terpenoid.

\section{Discussion}

Genome assembly assessment

The results of the five preliminary assemblies were evaluated using two approaches: BUSCO v3.0 completeness calculation [44] and CGAL assembly likelihood calculation [45]. Falcon assembly displayed the lowest number of scaffolds and the highest N50 value. However, it was the most incomplete assembly, as it lacked the highest number of BUSCO entries. This implies that this 


\section{a}

\section{Hypsin N-term 1 ITFQGDLDARQQVITNADTRRKRDVRAA 28} Hypma_04324 185 ITFQGCSPARQTVITNAITRARADVRAA 212

b

Hypma_04324 protein (354 aa)

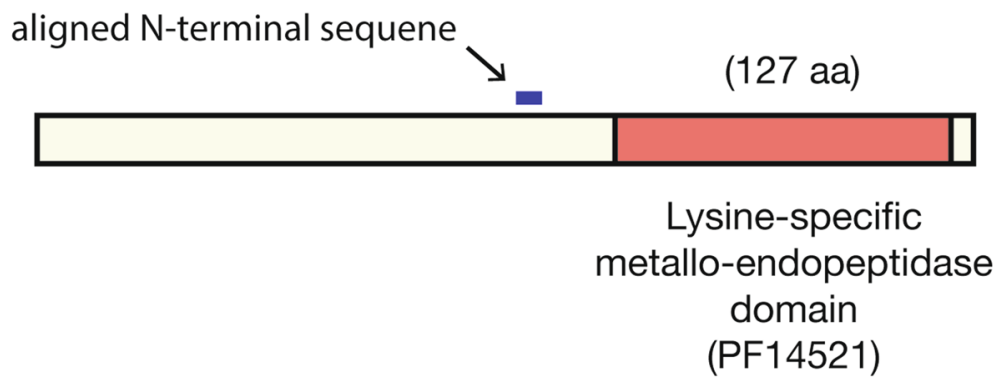

Fig. 4 A candidate hypsin gene obtained using sequence alignment. a Alignment of the experimentally determined $\mathrm{N}$-terminal sequence of hypsin and BLAST-searched Hypma_04324. b Schematic representation of the Hypma_04324

assembly misses some genomic regions that could contain protein-coding genes. Scaffolding with SSPACE-longread on Allpaths assembly decreased the number of scaffolds by nearly half, but the actual number of contigs increased. SPAdes using all three libraries generated comparable assemblies as the other libraries with the lowest CGAL value. In terms of genome completeness inferred by BUSCO, Allpaths+PBJelly displayed the best quality among the five candidates with the largest assembly size (42.7 Mbp). For further investigation, we predicted protein-coding genes in the Allpaths+PBJelly and Falcon assemblies. There were 571 more genes predicted in the Allpaths+PBJelly assembly than in the Falcon assembly (Additional file 2: Table S4).
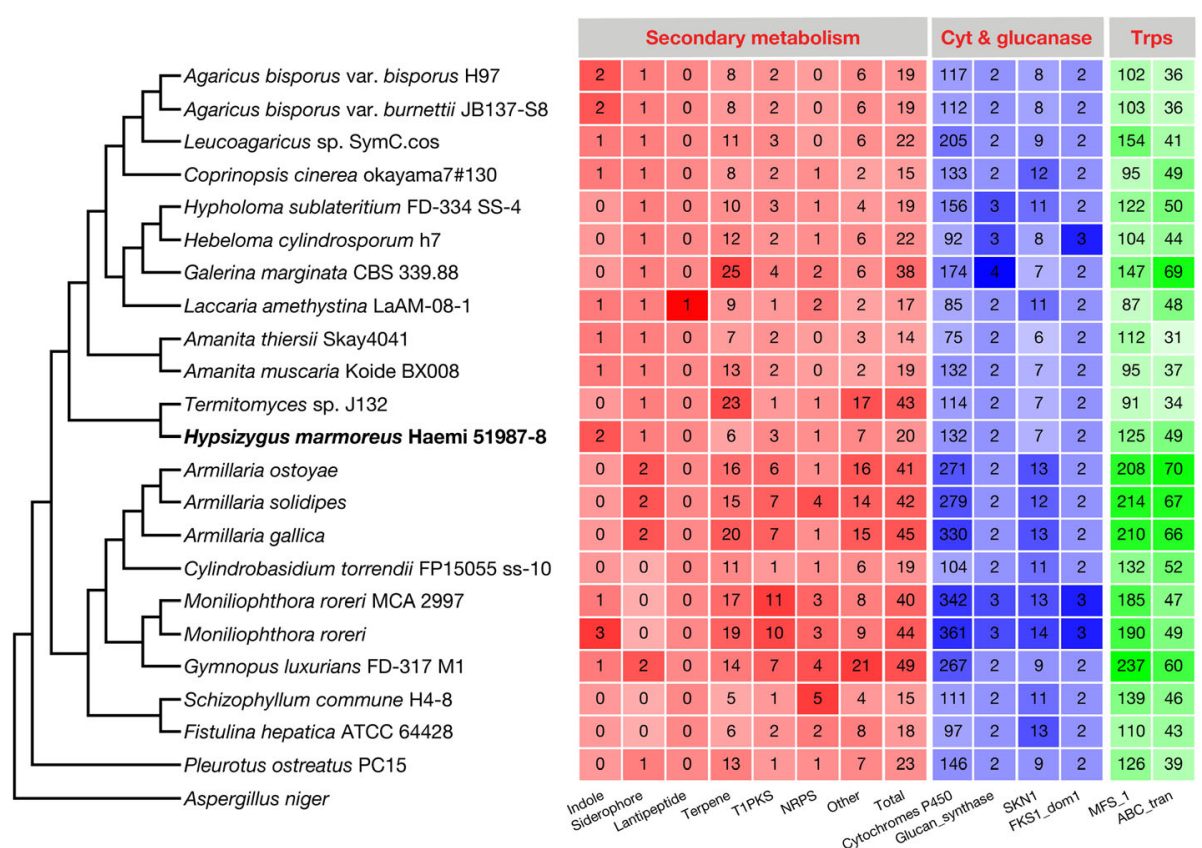

Fig. 5 Secondary metabolism genes of 22 Agaricales species. Detailed methods for building the genome tree and predicting secondary metabolism genes are described in the Methods section. The genomes are listed in Additional file 2: Table S2. Aspergillus niger was used as an outgroup 


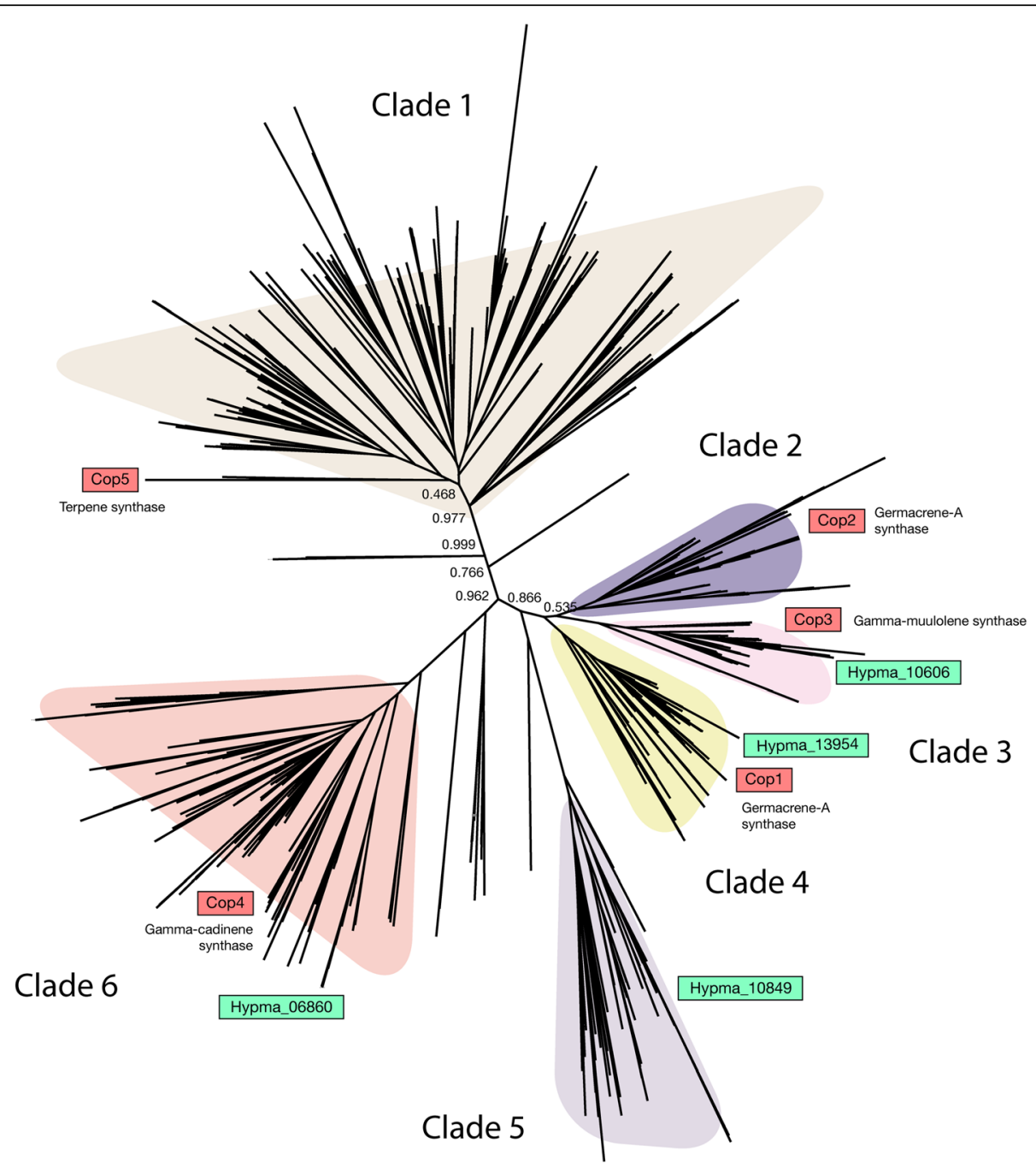

Fig. 6 A gene tree of terpene synthases. We used 759 putative terpene synthase genes from an orthologous group containing known terpene synthase genes

In particular, there were nine Pfam domains that were not found in the Falcon assembly gene prediction, which could affect downstream functional analyses. Sequence alignment revealed 1.27 Mbp and $782 \mathrm{kbp}$ of unique regions in the Allpaths+PBJelly and Falcon assemblies against each other, respectively. One unique region in the Allpaths+PBJelly assembly included a missing BUSCO entry. Although the Allpaths+PBJelly assembly was rather fragmented compared to the assemblies from the other methods, the Allpaths+PBJelly assembly contained more genomic regions, implying more completeness for functional and comparative analyses. Thus, we selected the Allpaths+PBJelly assembly as the final assembly for the subsequent analyses.

\section{Putative hypsin gene}

The putative hypsin gene had $71 \%$ identity to reported hypsin $\mathrm{N}$-terminal sequence. The discrepancy may be due to their regional origins, as the reported $H$. marmoreus was isolated from China whereas our sample was obtained from Korea. The matched region with the known N-terminal sequence was located starting at amino acid 185. The calculated weight of the truncated protein from the matched region was estimated as $18.2 \mathrm{kDa}$, which is similar to the reported molecular weight $(20 \mathrm{kDa})$ of hypsin. As the reported hypsin was purified from cell extracts, the hypsin gene identified in this genome might be post-translationally processed to an active form. Interestingly, the hypsin gene contained a lysine-specific metallo-endopeptidase (Pfam: PF14521, peptidase M35 family) domain at amino acids 223-349 but displayed no significant sequence similarity with alphamomorcharin or trichosanthin. This protein was predicted to have a signal peptide for secretion, similarly as other peptidase M35 family proteins. The $H$. marmoreus genome contained four copies of this protein, which is common in other Agaricomycetes. Specifically, 39 of 70 genomes contained at 


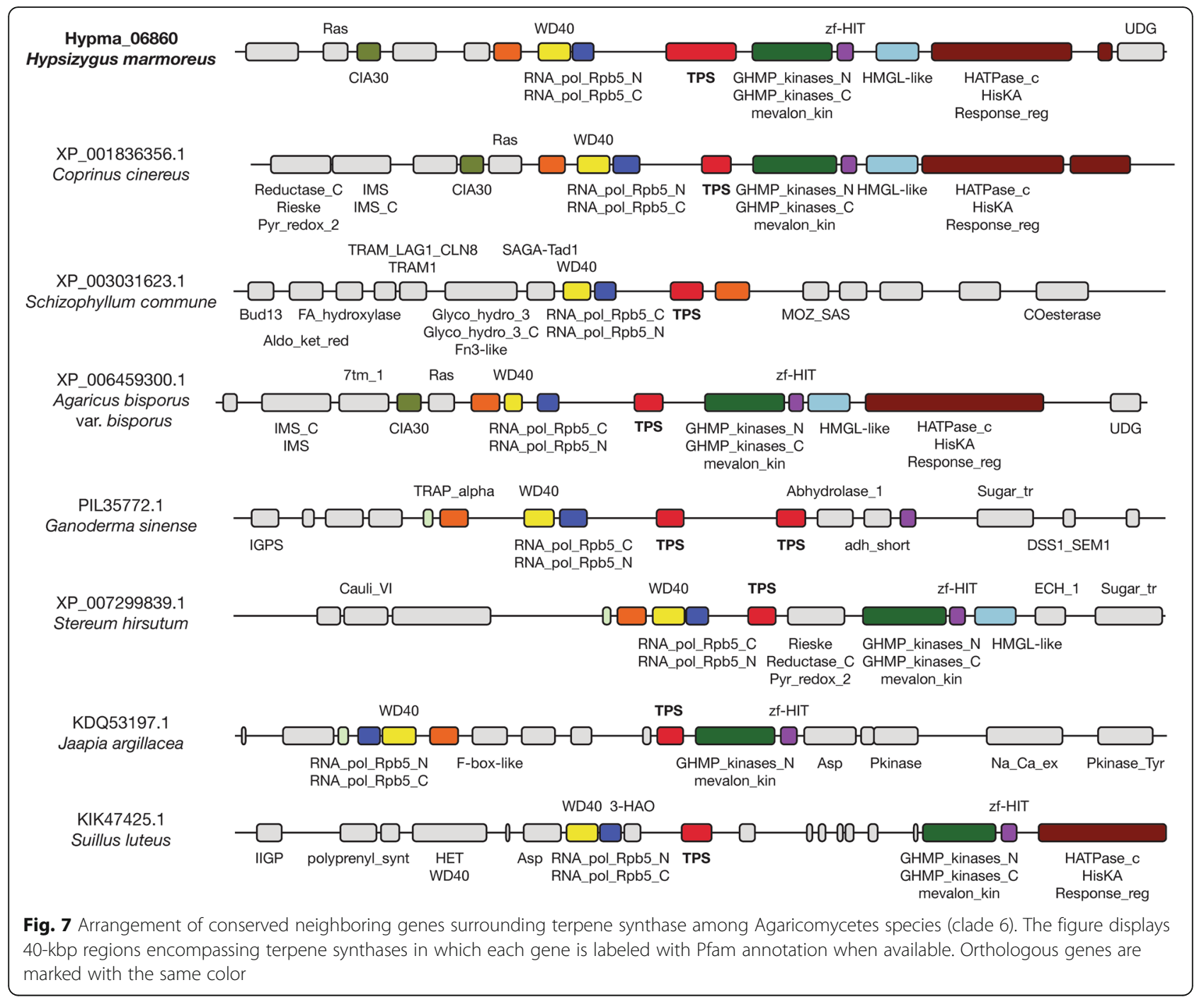

least one ortholog, and the Rhizoctonia solani genome had 38 copies. The exact molecular function and medicinal effects of this candidate hypsin remain to be elucidated. We were unable to find a candidate gene for another known bioactive protein, marmorin, in the current gene prediction and genome assembly.

\section{Conclusions}

We constructed a high-quality genome assembly and annotation for the genes and gene clusters of medicinal compounds. Thus, this study serves as a primary case study for combining experimental results and the genomics of mushrooms containing highly valuable bioactive compounds.

\section{Methods}

\section{Library preparation and sequencing}

The mycelium of $H$. marmoreus Haemi 51,987-8 (Korean Collection for Type Cultures No. 46454, http://
kctc.kribb.re.kr/En/Kctc.aspx) was cultured in $65 \%$ potato dextrose broth (BD Difco ${ }^{\text {TM }}$, Franklin Lakes, NJ, USA) under shaking at $24{ }^{\circ} \mathrm{C}$ for 3-7 days. The genomic DNA of the monokaryotic strain was extracted from mycelia using a DNeasy Plant Mini Kit (Qiagen, Valencia, CA, USA). Three libraries were generated for genome assembly: paired-end and mate-pair Illumina libraries and a PacBio library. In total, 40 and $9 \mathrm{Gbp}$ of data were generated using Illumina and PacBio, respectively. RNA molecules were extracted from mycelia using an RNeasy Plant Mini Kit (Qiagen). Two mRNA sequencing libraries were generated for gene prediction (23 Gbp). Illumina reads were trimmed and filtered by base quality and read length using HTQC 1.92.3 [46], and PacBio reads were filtered by read length and read quality using SMRT analysis 2.3.0 RS_Subreads protocol (https:// www.pacb.com/products-and-services/analytical-software/smrt-analysis/). The sequenced data are summarized in Table 1. 


\section{Genome size estimation}

The genome size of $H$. marmoreus was estimated using k-mer frequency. The paired-end Illumina library was used to draw k-mer frequency plots, in which 17 and 19 k-mers, respectively, were set. JellyFish 2.2.4 [47] was used to calculate the frequencies.

\section{Genome assembly}

Using three different genomic DNA libraries (Illumina paired-end, Illumina mate-pair, and PacBio), we built five candidate assemblies using the following strategies: (i) Allpaths, (ii) Allpaths+PBJelly, (iii) Allpaths+SSPACE-longread, (iv) Falcon, and (v) SPAdes. First, Illumina paired-end and mate-pair libraries were used to run Allpaths [48] with PLOIDY $=1$. This assembly was improved by running PBJelly 15.8.24 [17] and SSPACE-longread v1.1 [18], which both require filtered subreads (filtered PacBio reads) as an input. Falcon [20] was run using only PacBio reads with a cutoff length of $8000 \mathrm{bp}$. FinisherSC [21] and Quiver [22] were run to improve the Falcon assembly. We also used SPAdes v3.10.1 [19] for a hybrid assembly using all three libraries with the --careful option. The five assembly candidates were evaluated using BUSCO 3.0.2 [44] with basidiomycota_odb9 lineage data and CGAL 0.9.6 [45].

\section{Post-process of the assembly}

We checked whether the assembly contained mitochondrial or contaminated sequences. In a fungal genome assembly, the mitochondrial genome generally has a much higher sequence depth and a lower GC content than the nuclear genome [49]. We ran BLASTn with the assembly against the NCBI mitochondrial genome database (ftp://ftp.ncbi.nlm.nih.gov/blast/db/FASTA/mito.nt.gz). Hit scaffolds were investigated for their read coverages and GC contents if they were outliers relative to other scaffolds. Sequence contamination was checked using Blobology 2015-12-16 [50].

\section{Genome annotation}

We first detected repeat regions to mask before gene prediction using RepeatModeler and RepeatMasker (http:// www.repeatmasker.org). Protein-encoding genes in the assembly were predicted using the FunGAP pipeline. mRNA sequences were sampled into 58.8 million reads (5.83 billon bases) to decrease the computing time. mRNA reads were mapped into the genome using Hisat 2.0.2 [51]. The mapped reads were assembled using Trinity 2.2.0 [52]. Laccaria bicolor was set as the Augustus [24] species model. Noncoding RNA elements such as tRNAs, snoRNAs, and microRNAs were annotated by scanning Rfam database release 12.1 [53] using Infernal 1.1.1 [54].

\section{Reference genomes for comparative analysis}

We downloaded the protein sequences of 69 Agaricomycetes species, including $H$. marmoreus, in FASTA format from the
NCBI database. We ran OrthoFinder 1.0.6 [55] to obtain orthologous genes from the genomes and selected 102 single-copy orthologs to build a species tree. The program was run with Aspergillus niger protein sequences comprising an outgroup (GenBank accession: GCF_000002855.3). RAxML 7.3.0 [56] was used to build the tree with " $f a-x$ 12345 -p 12345 -\# 100 -m PROTGAMMAWAG” options. The tree was visualized using Dendroscope 3.5.9 [57].

\section{CAZyme analysis}

CAZymes in the H. marmoreus genome and the 69 reference genomes were identified by combining dbCAN, BLASTp, and Pfam domain predictions. The dbCAN 5.0 database [58] was searched using hmmscan [59] with default options, and the result was parsed using a script (http://cys.bios.niu.edu/dbCAN/download/hmmscan-parser.sh). BLASTp was run against CAZyme protein sequences download from dbCAN (http://cys.bios.niu.edu/ dbCAN/download/CAZyDB.03172015.fa) with an E-value cutoff of $1 \mathrm{e}-10$. For Pfam domain prediction, we ran InterProScan 5.25-64.0 [60] against the Pfam 31.0 database and extracted CAZyme domain-containing proteins. Pfam-A.full data was used to obtain Pfam domains associated with CAZymes. We annotated CAZymes when they were predicted identically by more than two methods and added Pfam and dbCAN-only predicted CAZymes after manual curation. Enriched or depleted CAZymes were estimated using Fisher's exact test from the Python Scipy package (https://www.scipy.org/).

\section{Search for hypsin and marmorin}

The N-terminal sequences of hypsin and marmorin, namely "ITFQGDLDARQQVITNADTRRKRDVRAA" and "AEGTL LGSRATCESGNSMY," respectively, were retrieved from previous publications $[5,6]$. BLASTp was used against all protein sequences. We also ran tBLASTn against the assembled transcripts and genome assembly for unannotated genes.

\section{Predicting secondary metabolism genes}

AntiSMASH 4.0.1 [61] was used to predict secondary metabolism genes in the genomes. The annotation of secondary metabolism genes was based on Pfam notation as follows: cytochrome P450, PF00067; glucan synthase, PF02364, PF03935, and PF14288; and transporters, PF07690 and PF00005. To obtain the terpene synthase genes of 70 Agaricomycetes genomes, we selected an ortholog group in which known terpene synthase genes (Cop1-5 genes) are included. The ortholog groups were estimated using OrthoFinder 1.0.6 [55]. The group contained 759 gene members. Seventeen incomplete genes (not starting with methionine) were excluded. The resulting 742 genes were used to build a gene tree using Mafft 7.273 [62] and FastTree 2.1.3 [63] for sequence alignment and tree building, respectively. 


\section{Additional files}

Additional file 1: Figure S1. K-mer frequency of genomic reads. Figure S2. Sequence coverage histogram. Figure S3. Read coverage and GC content plot. Figure S4. Genome sizes and gene numbers of Agaricomycetes. Figure S5. Terpene synthase genes of 70 Agraicomycetes. Figure S6. The gene tree of terpene synthase genes of ten Agraicomycetes. Figure S7. Arrangement of conserved neighboring genes surrounding terpene synthase among Agaricomycetes (clade 3). Figure S8. Arrangement of conserved neighboring genes surrounding terpene synthase among Agaricomycetes (clade 4). Figure S9. Arrangement of conserved neighboring genes surrounding terpene synthase among Agaricomycetes (clade 5). Figure S10. Transcriptional expression of terpene synthase genes and their neighboring genes. (PDF $2772 \mathrm{~kb}$ )

Additional file 2: Table S1. Gene predictions of various programs. Table S2. Reference genomes for comparative analysis. Table S3. Secondary metabolism genes in the 70 Agaricomycetes genomes. Table S4. Gene predictions of genome assemblies generated by different methods. (XLSX $29 \mathrm{~kb}$ )

\section{Abbreviations}

AA: Auxiliary activity; CAZyme: Carbohydrate active enzyme; CBM: Carbohydrate-binding module; CE: Carbohydrate esterase; GH: Glycoside hydrolase; GHMP kinase: Galacto-kinase, homoserine-kinase, mevalonate-kinase, phosphomevalonate-kinase; NCBI: National Center for Biotechnology Information

\section{Acknowledgments}

The authors would like to thank School of Life Sciences and Biotechnology for BK21 PLUS, Korea University for processing charge in open access journals.

\section{Funding}

This work was conducted with the support of the Cooperative Research Program for the National Agricultural Genome Program (Project No. PJ01044003) of the Rural Development Administration; New and Renewable Energy Core Technology Program of the Korea Institute of Energy Technology Evaluation and Planning grants from the Ministry of Trade, Industry and Energy (No. 20173010092460); and a Korea University Grant. The funders had no role in study design, data collection and interpretation, or the decision to submit the work for publication.

\section{Availability of data and materials}

Genomic sequence and annotation data supporting the findings of this study have been deposited in GenBank with the primary accession code LUEZ00000000. The version described in this paper is LUEZ02000000. The DNA and RNA sequences were deposited into the NCBI sequence read archive (SRA) with accessions SRR7874780-SRR7874789.

\section{Authors' contributions}

SK, YO, WK, HP, HC, KJ, and JK isolated and identified the strains; SK, YO, WK, $\mathrm{HP}, \mathrm{HC}, \mathrm{KJ}, \mathrm{JK}$ and IC designed and performed the experiments; SK, YO, WK, $\mathrm{HP}, \mathrm{HC}, \mathrm{KJ}$, and JK contributed to data acquisition; BM, SK, JK and IC performed bioinformatics and statistical analyses; and BM, SK, JK and IC prepared the manuscript. All authors edited and commented on the manuscript. All authors have read and approved the final manuscript.

\section{Ethics approval and consent to participate}

Not applicable.

\section{Consent for publication}

Not applicable.

\section{Competing interests}

The authors declare that they have no competing interests.

\section{Publisher's Note}

Springer Nature remains neutral with regard to jurisdictional claims in published maps and institutional affiliations.

\section{Author details}

'Department of Biotechnology, College of Life Sciences and Biotechnology, Korea University, 145 Anam-ro, Seongbuk-Gu, Seoul 02841, Korea. ²Genomics Division, National Institute of Agricultural Sciences, Rural Development Administration (RDA), Jeonju 54874, Korea. ${ }^{3}$ Mushroom Division, National Institute of Horticultural and Herbal Science (NHHS), Rural Development Administration (RDA), Eumseong 27709, Korea.

Received: 7 June 2018 Accepted: 11 October 2018

Published online: 01 November 2018

\section{References}

1. Saitoh H, Feng W, Matsuzawa T, Ikekawa T. Antitumor activity of Hypsizigus marmoreus. II. Preventive effect against lung metastasis of Lewis lung carcinoma. Yakugaku Zasshi. 1997;117(12):1006-10.

2. Chang JS, Son JK, Li G, Oh EJ, Kim JY, Park SH, Bae JT, Kim HJ, Lee IS, Kim $\mathrm{OM}$, et al. Inhibition of cell cycle progression on HepG2 cells by hypsiziprenol A9, isolated from Hypsizigus marmoreus. Cancer Lett. 2004; 212(1):7-14

3. Puri M, Kaur I, Perugini MA, Gupta RC. Ribosome-inactivating proteins: current status and biomedical applications. Drug Discov Today. 2012;17(1314):774-83.

4. Yang W, Shi J, Yang B. Antibacterial characteristics of the liquid cell-free culture of Hypsizigus marmoreus. J Northwest A \& F Univ-Natural Sci Edition. 2009;37(6):194-8.

5. Lam SK, Ng TB. Hypsin, a novel thermostable ribosome-inactivating protein with antifungal and antiproliferative activities from fruiting bodies of the edible mushroom Hypsizigus marmoreus. Biochem Biophys Res Commun. 2001;285(4):1071-5

6. Wong $\mathrm{JH}$, Wang $\mathrm{HX}, \mathrm{Ng} \mathrm{TB}$. Marmorin, a new ribosome inactivating protein with antiproliferative and HIV-1 reverse transcriptase inhibitory activities from the mushroom Hypsizigus marmoreus. Appl Microbiol Biotechnol. 2008:81(4):669-74

7. Jeong YT, Yang BK, Jeong SC, Kim SM, Song CH. Ganoderma applanatum: a promising mushroom for antitumor and immunomodulating activity. Phytother Res. 2008;22(5):614-9.

8. Agger S, Lopez-Gallego F, Schmidt-Dannert C. Diversity of sesquiterpene synthases in the basidiomycete Coprinus cinereus. Mol Microbiol. 2009;72(5): 1181-95.

9. Wawrzyn GT, Quin MB, Choudhary S, Lopez-Gallego F, Schmidt-Dannert C Draft genome of Omphalotus olearius provides a predictive framework for sesquiterpenoid natural product biosynthesis in Basidiomycota. Chem Biol. 2012;19(6):772-83.

10. Quin MB, Flynn CM, Wawrzyn GT, Choudhary S, Schmidt-Dannert C. Mushroom hunting by using bioinformatics: application of a predictive framework facilitates the selective identification of sesquiterpene synthases in basidiomycota. Chembiochem. 2013;14(18):2480-91.

11. Xu X, Yan H, Chen J, Zhang X. Bioactive proteins from mushrooms. Biotechnol Adv. 2011;29(6):667-74.

12. Wang $\mathrm{H}, \mathrm{Ng}$ TB. Isolation and characterization of velutin, a novel lowmolecular-weight ribosome-inactivating protein from winter mushroom (Flammulina velutipes) fruiting bodies. Life Sci. 2001;68(18):2151-8.

13. Wang HX, Ng TB. Flammulin: a novel ribosome-inactivating protein from fruiting bodies of the winter mushroom Flammulina velutipes. Biochem Cell Biol. 2000;78(6):699-702.

14. Lam SK, Ng TB. First simultaneous isolation of a ribosome inactivating protein and an antifungal protein from a mushroom (Lyophyllum shimeji) together with evidence for synergism of their antifungal effects. Arch Biochem Biophys. 2001;393(2):271-80.

15. Kurt A, Fukuta Y, Mori M, Kishimoto N, Shirasaka N. Draft genome sequence of the Basidiomycetous fungus Flammulina velutipes TR19. Genome Announc. 2016;4(3):e00505-16

16. Park YJ, Baek JH, Lee S, Kim C, Rhee H, Kim H, Seo JS, Park HR, Yoon DE, Nam JY, et al. Whole genome and global gene expression analyses of the model mushroom Flammulina velutipes reveal a high capacity for lignocellulose degradation. PLoS One. 2014;9(4):e93560.

17. English AC, Richards S, Han Y, Wang M, Vee V, Qu J, Qin X, Muzny DM, Reid JG, Worley KC, et al. Mind the gap: upgrading genomes with Pacific biosciences RS long-read sequencing technology. PLoS One. 2012;7(11): e47768. 
18. Boetzer M, Pirovano W. SSPACE-LongRead: scaffolding bacterial draft genomes using long read sequence information. BMC Bioinformatics. 2014; 15:211.

19. Bankevich A, Nurk S, Antipov D, Gurevich AA, Dvorkin M, Kulikov AS, Lesin VM, Nikolenko SI, Pham S, Prjibelski AD, et al. SPAdes: a new genome assembly algorithm and its applications to single-cell sequencing. J Comput Biol. 2012;19(5):455-77.

20. Chin CS, Peluso P, Sedlazeck FJ, Nattestad M, Concepcion GT, Clum A, Dunn C, O'Malley R, Figueroa-Balderas R, Morales-Cruz A, et al. Phased diploid genome assembly with single-molecule real-time sequencing. Nat Methods. 2016;13(12):1050-4.

21. Lam KK, LaButti K, Khalak A, Tse D. FinisherSC: a repeat-aware tool for upgrading de novo assembly using long reads. Bioinformatics (Oxford, England). 2015;31(19):3207-9

22. Chin CS, Alexander DH, Marks P, Klammer AA, Drake J, Heiner C, Clum A, Copeland A, Huddleston J, Eichler EE, et al. Nonhybrid, finished microbial genome assemblies from long-read SMRT sequencing data. Nat Methods. 2013;10(6):563-9.

23. Min B, Grigoriev IV, Choi IG. FunGAP: Fungal Genome Annotation Pipeline using evidence-based gene model evaluation. Bioinformatics (Oxford, England). 2017;33(18):2936-7.

24. Stanke M, Morgenstern B. AUGUSTUS: a web server for gene prediction in eukaryotes that allows user-defined constraints. Nucleic Acids Res. 2005; 33(Web Server):W465-7.

25. Hoff KJ, Lange S, Lomsadze A, Borodovsky M, Stanke M. BRAKER1: unsupervised RNA-Seq-based genome annotation with GeneMark-ET and AUGUSTUS. Bioinformatics (Oxford, England). 2016;32(5):767-9.

26. Cantarel BL, Korf I, Robb SM, Parra G, Ross E, Moore B, Holt C, Sanchez Alvarado A, Yandell M. MAKER: an easy-to-use annotation pipeline designed for emerging model organism genomes. Genome Res. 2008;18(1):188-96.

27. Gupta DK, Ruhl M, Mishra B, Kleofas V, Hofrichter M, Herzog R, Pecyna MJ, Sharma R, Kellner $\mathrm{H}$, Hennicke $\mathrm{F}$, et al. The genome sequence of the commercially cultivated mushroom Agrocybe aegerita reveals a conserved repertoire of fruiting-related genes and a versatile suite of biopolymerdegrading enzymes. BMC Genomics. 2018;19(1):48.

28. Yuan Y, Wu F, Si J, Zhao YF, Dai YC. Whole genome sequence of Auricularia heimuer (Basidiomycota, Fungi), the third most important cultivated mushroom worldwide. Genomics. 2017. https://doi.org/10.1016/j.ygeno. 2017.12.013

29. Lombard V, Golaconda Ramulu H, Drula E, Coutinho PM, Henrissat B. The carbohydrate-active enzymes database (CAZy) in 2013. Nucleic Acids Res. 2014:42(Database issue):D490-5.

30. Krupodorova T, Ivanova T, Barshteyn V. Screening of extracellular enzymatic activity of macrofungi. J Microbiol Biotechnol Food Sci. 2014;3(4):315.

31. Riley R, Salamov AA, Brown DW, Nagy LG, Floudas D, Held BW, Levasseur A, Lombard V, Morin E, Otillar R, et al. Extensive sampling of basidiomycete genomes demonstrates inadequacy of the white-rot/brown-rot paradigm for wood decay fungi. Proc Natl Acad Sci. 2014;111(27):9923-8.

32. Hirabayashi J, Dutta SK, Kasai K. Novel galactose-binding proteins in Annelida. Characterization of 29-kDa tandem repeat-type lectins from the earthworm Lumbricus terrestris. J Biol Chem. 1998:273(23):14450-60.

33. Hazes B, Read RJ. A mosquitocidal toxin with a ricin-like cell-binding domain. Nat Struct Biol. 1995:2(5):358-9.

34. Hazes B. The (QxW)3 domain: a flexible lectin scaffold. Protein Sci. 1996;5(8): 1490-501.

35. Ng TB, Liu WK, Sze SF, Yeung HW. Action of alpha-momorcharin, a ribosome inactivating protein, on cultured tumor cell lines. Gen Pharmacol. 1994;25(1):75-7

36. Li MX, Yeung HW, Pan LP, Chan SI. Trichosanthin, a potent HIV-1 inhibitor, can cleave supercoiled DNA in vitro. Nucleic Acids Res. 1991;19(22):6309-12.

37. Hashimoto M, Nonaka T, Fujii I. Fungal type III polyketide synthases. Nat Prod Rep. 2014;31(10):1306-17.

38. Acimovic J, Goyal S, Kosir R, Golicnik M, Perse M, Belic A, Urlep Z, Guengerich FP, Rozman D. Cytochrome P450 metabolism of the postlanosterol intermediates explains enigmas of cholesterol synthesis. Sci Rep. 2016;6:28462.

39. Perlin MH, Andrews J, Toh SS. Essential letters in the fungal alphabet: $A B C$ and MFS transporters and their roles in survival and pathogenicity. Adv Genet. 2014;85:201-53.

40. Andreassi $J \mathrm{~L} 2 \mathrm{nd}$, Leyh TS. Molecular functions of conserved aspects of the GHMP kinase family. Biochemistry. 2004;43(46):14594-601.
41. Bach TJ. Some new aspects of isoprenoid biosynthesis in plants--a review. Lipids. 1995:30(3):191-202.

42. Bragantini B, Tiotiu D, Rothe B, Saliou JM, Marty H, Cianferani S, Charpentier B, Quinternet M, Manival X. Functional and structural insights of the zincfinger HIT protein family members involved in box C/D snoRNP biogenesis. J Mol Biol. 2016;428(11):2488-506.

43. Perego M, Hoch JA. Protein aspartate phosphatases control the output of twocomponent signal transduction systems. Trends Genet. 1996;12(3):97-101.

44. Simao FA, Waterhouse RM, loannidis P, Kriventseva EV, Zdobnov EM. BUSCO: assessing genome assembly and annotation completeness with single-copy orthologs. Bioinformatics (Oxford, England). 2015;31(19):3210-2.

45. Rahman A, Pachter L. CGAL: computing genome assembly likelihoods. Genome Biol. 2013;14(1):R8.

46. Yang X, Liu D, Liu F, Wu J, Zou J, Xiao X, Zhao F, Zhu B. HTQC: a fast quality control toolkit for Illumina sequencing data. BMC Bioinformatics. 2013;14:33.

47. Marcais G, Kingsford C. A fast, lock-free approach for efficient parallel counting of occurrences of k-mers. Bioinformatics (Oxford, England). 2011; 27(6):764-70.

48. Butler J, MacCallum I, Kleber M, Shlyakhter IA, Belmonte MK, Lander ES, Nusbaum C, Jaffe DB. ALLPATHS: de novo assembly of whole-genome shotgun microreads. Genome Res. 2008;18(5):810-20.

49. Clum A. Genome Assembly. Methods Mol Biol (Clifton, NJ). 2018;1775:141-53.

50. Kumar S, Jones M, Koutsovoulos G, Clarke M, Blaxter M. Blobology: exploring raw genome data for contaminants, symbionts and parasites using taxonannotated GC-coverage plots. Front Genet. 2013;4:237.

51. Kim D, Langmead B, Salzberg SL. HISAT: a fast spliced aligner with low memory requirements. Nat Methods. 2015;12(4):357-60.

52. Grabherr MG, Haas BJ, Yassour M, Levin JZ, Thompson DA, Amit I, Adiconis X, Fan L, Raychowdhury R, Zeng Q, et al. Full-length transcriptome assembly from RNA-Seq data without a reference genome. Nat Biotechnol. 2011;29(7): 644-52.

53. Nawrocki EP, Burge SW, Bateman A, Daub J, Eberhardt RY, Eddy SR, Floden EW, Gardner PP, Jones TA, Tate J, et al. Rfam 12.0: updates to the RNA families database. Nucleic Acids Res. 2015;43(Database issue):D130-7.

54. Nawrocki EP, Eddy SR. Infernal 1.1: 100-fold faster RNA homology searches. Bioinformatics (Oxford, England). 2013;29(22):2933-5.

55. Emms DM, Kelly S. OrthoFinder: solving fundamental biases in whole genome comparisons dramatically improves orthogroup inference accuracy. Genome Biol. 2015;16:157.

56. Stamatakis A. RAxML version 8: a tool for phylogenetic analysis and postanalysis of large phylogenies. Bioinformatics (Oxford, England). 2014;30(9): 1312-3.

57. Huson DH, Scornavacca C. Dendroscope 3: an interactive tool for rooted phylogenetic trees and networks. Syst Biol. 2012;61(6):1061-7.

58. Yin Y, Mao X, Yang J, Chen X, Mao F, Xu Y. dbCAN: a web resource for automated carbohydrate-active enzyme annotation. Nucleic Acids Res. 2012 40(Web Server issue):W445-51.

59. Finn RD, Clements J, Eddy SR. HMMER web server: interactive sequence similarity searching. Nucleic Acids Res. 2011;39(Web Server issue):W29-37.

60. Jones $\mathrm{P}$, Binns D, Chang HY, Fraser M, Li W, McAnulla C, McWilliam H, Maslen J, Mitchell A, Nuka G, et al. InterProScan 5: genome-scale protein function classification. Bioinformatics (Oxford, England). 2014;30(9):1236-40.

61. Blin K, Wolf T, Chevrette MG, Lu X, Schwalen CJ, Kautsar SA, Suarez Duran HG, de Los Santos ELC, Kim HU, Nave M, et al. antiSMASH 4.0-improvements in chemistry prediction and gene cluster boundary identification. Nucleic Acids Res. 2017;45(W1):W36-W41

62. Katoh K, Standley DM. MAFFT multiple sequence alignment software version 7: improvements in performance and usability. Mol Biol Evol. 2013; 30(4):772-80.

63. Price MN, Dehal PS, Arkin AP. FastTree 2--approximately maximumlikelihood trees for large alignments. PLoS One. 2010;5(3):e9490. 